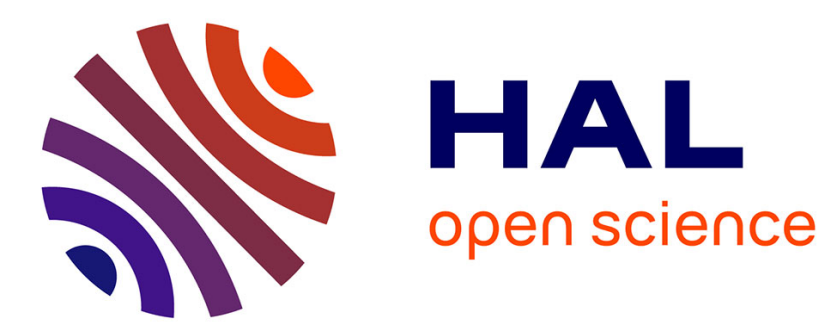

\title{
Schwarz method for dual contact problems
}

Lori Badea, Frédéric Lebon

\section{To cite this version:}

Lori Badea, Frédéric Lebon. Schwarz method for dual contact problems. Computational \& Applied Mathematics, 2017, 36 (1), pp.719-731. 10.1007/s40314-015-0255-y . hal-01508775

\section{HAL Id: hal-01508775 \\ https://hal.science/hal-01508775}

Submitted on 20 May 2018

HAL is a multi-disciplinary open access archive for the deposit and dissemination of scientific research documents, whether they are published or not. The documents may come from teaching and research institutions in France or abroad, or from public or private research centers.
L'archive ouverte pluridisciplinaire HAL, est destinée au dépôt et à la diffusion de documents scientifiques de niveau recherche, publiés ou non, émanant des établissements d'enseignement et de recherche français ou étrangers, des laboratoires publics ou privés. 


\title{
Schwarz method for dual contact problems
}

\author{
Lori Badea $^{1}$ • Frédéric Lebon ${ }^{2}$
}

\begin{abstract}
In this paper, we analyze the convergence of the Schwarz method for contact problems with Tresca friction formulated in stress variables. In this dual variable, the problem is written as a variational inequality in the space $H_{\text {div }}(\Omega), \Omega$ being the domain of the problem. The method is introduced as a subspace correction algorithm. In this case, the global convergence and the error estimation of the method are already proved in the literature under some assumptions. However, the checking of these hypotheses in the space $H_{\text {div }}(\Omega)$ cannot be proved easily, as for the space $H^{1}(\Omega)$. The main result of this paper is to prove that these hypotheses are verified for this particular variational inequality. As in the case of the classical problems formulated in primal variables, the error estimate we obtain depends on the overlapping parameter of the domain decomposition.
\end{abstract}

Keywords Contact problems - Dual formulation - Domain decomposition methods · Schwarz method $\cdot$ Subspace correction methods $\cdot$ Variational inequalities

Mathematics Subject Classification $65 \mathrm{~N} 55 \cdot 65 \mathrm{~K} 15 \cdot 74 \mathrm{M} 10 \cdot 74 \mathrm{M} 15$

\section{Introduction}

Traditionally, frictional contact problems are formulated in terms of the displacements (primal variables) (Cocu 1984; Raous et al. 1988; Cocou et al. 1996; Lebon 2003) or of forces-

Communicated by Hector Ramirez.

Frédéric Lebon

lebon@1ma.cnrs-mrs.fr

Lori Badea

lori.badea@imar.ro

1 Institute of Mathematics of the Romanian Academy, P.O. Box 1-764, 014700 Bucharest, Romania

2 LMA, CNRS UPR 7051, Aix-Marseille Université, Centrale Marseille, 31 chemin Joseph Aiguier, 13402 Marseille Cedex 20, France 
displacements (mixed variables) (Alart and Curnier 1991; Alart and Lebon 1995; Wriggers 2002). There also exists a third kind of formulation in terms of stresses (dual variables) (Telega 1991). This formulation has given numerical developments in the last few years (Bisegna et al. 2001). This approach is little used but it has a lot of advantages in point of view of numerical computation. The first advantage is that the stress tensor is the variable of interest in the mechanical design and is directly obtained by this formulation. Another one is the capability of deriving an a posteriori error estimator (Kuss and Lebon 2011). Also, in the dual case, the problem is formulated as a variational inequality (of the first kind) which is more suitable for the numerical point of view than the variational inequality of the second kind in the primal case which contains a non-differentiable term.In addition, the numerical experiments have shown that the linear systems in dual variable are better conditioned than those in primal variables (Bisegna et al. 2004). On the other hand, this approach is less used than the primal or mixed ones because of numerical difficulties related to the finite elements which have to be used (Kuss and Lebon 2009).

In the case of the Schwarz method, one of the difficulties is even the formulation of the method. In the primal case, the displacements are thought in $H^{1}$ and they have traces on the boundary of the domain, and the Schwarz method transfers, during the iteration, the solution values on a subdomain toward the solution on the neighboring subdomains. But, the dual approach utilizes the space $H_{\text {div }}$ where only the normal trace of the stresses exists. This difficulty has been avoided by interpreting the Schwarz method as a subspace correction method. In this case, we have to find corrections in appropriate subspaces which may not have any connection with the notion of trace.

We prove in this paper that, for the weak formulation of the dual contact problem with Tresca friction, the Schwarz method converges and also gives an error estimate depending on the domain decomposition parameter. It is well known that the solution of the contact problem with Coulomb friction can be obtained by a fixed point procedure in which we solve a contact problem with Tresca friction at each iteration step (Licht et al. 1991). The study of the method for the discretized problem by finite elements, the one-level or multi-level methods, will be treated in a subsequent paper.

The paper is organized as follows. In Sect. 2, the mechanical and the weak formulations of the problem in dual variables are introduced. Section 3 is dedicated to the study of the Schwarz algorithm. As we said above, using the primal variables, the problem is formulated as a variational inequality of the second kind which contains a non-differentiable term. Oneand two-level Schwarz methods for such formulations of the frictional contact problems have been given in Badea and Krause (2012). When the problem is formulated in dual variables, we get a simply variational inequality (of the first kind) and we shall use the results in Badea (2003) to prove the convergence of the method. For an abstract theory of Schwarz methods we recommend (Toselli and Widlund 2005). The algorithm is introduced as a subspace correction method and we rewrite a general convergence theorem from Badea (2003) in terms of the considered framework. We prove that the assumption made in the convergence theorem is verified and derive the constants in the error estimation. Finally, summing up the previous results we show that the method is geometrically convergent with a convergence rate which depends on the domain decomposition parameter.

\section{Statement of the problem}

We consider an elastic body occupying the open bounded set $\Omega \subset \mathbb{R}^{d},(d=2,3)$, with sufficiently smooth boundary. The boundary is divided in three open parts $\Gamma_{D}, \Gamma_{F}$ and $\Gamma_{C}$ such that 
$\partial \Omega=\bar{\Gamma}_{D} \cup \bar{\Gamma}_{F} \cup \bar{\Gamma}_{C}$ and we assume that the measure of $\Gamma_{D}$ is positive, meas $\left(\Gamma_{D}\right)>0$. We denote $u=\left(u_{i}\right)_{1 \leq i \leq d}$ the displacement field, $\varepsilon=\left(\varepsilon_{i j}(u)\right)_{1 \leq i, j \leq d}, \varepsilon_{i j}(u)=\frac{1}{2}\left(u_{i, j}+u_{j, i}\right)$, the linearized strain tensor and $\sigma=\left(\sigma_{i j}(u)\right)_{1 \leq i, j \leq d}, \sigma_{i j}(u)=\sum_{k, l=1}^{d} A_{i j k l} \varepsilon_{k l}(u)$ the Cauchy stress tensor. Operator $A$ is bounded and verifies usual symmetry and positivity conditions. We denote $S=A^{-1}$ the compliance tensor. This tensor has the following properties of symmetry, $S_{i j k l}=S_{k l i j}=S_{j i l k}$, and of positivity, i.e. there exists $\alpha>0$ such that $\sum_{i, j=1}^{d} \sum_{k, l=1}^{d} S_{i j k l} \tau_{i j} \tau_{k l} \geq \alpha \sum_{i, j=1}^{d} \tau_{i j} \tau_{i j}$.

The body is in receding contact with a rigid obstacle on the part of the boundary $\Gamma_{C}$. The contact is with friction and is represented by the unilateral contact law of Tresca.

On the contact boundary, displacement and stress vector are decomposed introducing the external normal unit vector $n$ to $\Gamma$ :

$$
\begin{gathered}
u_{N}=u \cdot n, \quad u_{T}=u-u_{N} \cdot n \\
\sigma_{N}=(\sigma n) \cdot n, \quad \sigma_{T}=\sigma n-\sigma_{N} n
\end{gathered}
$$

The body is submitted to a body force density $f$ in $\Omega$ and surface force density $g$ on $\Gamma_{F}$. We suppose $f \in\left(L^{2}(\Omega)\right)^{d}$ and $g \in\left(L^{2}\left(\Gamma_{F}\right)\right)^{d}$. On $\Gamma_{D}$ the displacement is given. Classically, the mechanical problem of an elastic body in Coulomb frictional contact with a rigid obstacle is given by:

Problem (P) Find the displacement field $u$ and the stress field $\sigma$ such that

$$
\begin{cases}\sigma=A \varepsilon(u) & \text { in } \Omega \\
\operatorname{div} \sigma+f=0 & \text { in } \Omega \\
u=u_{0} & \text { on } \Gamma_{D} \\
\sigma \cdot n=g & \text { on } \Gamma_{F} \\
u_{N} \leq 0, \sigma_{N} \leq 0, u_{N} \sigma_{N}=0 & \text { on } \Gamma_{C} \\
\left|\sigma_{T}\right| \leq-\mu \sigma_{N}^{*} & \text { on } \Gamma_{C} \text { and }\left\{\begin{array}{l}
\left|\sigma_{T}\right|<-\mu \sigma_{N}^{*} \Rightarrow u_{T}=0 \\
\left|\sigma_{T}\right|=-\mu \sigma_{N}^{*} \Rightarrow \exists \lambda \geq 0, u_{T}=-\lambda \sigma_{T}
\end{array}\right.\end{cases}
$$

where $\mu$ is the friction coefficient (bounded and positive) and ${ }^{*}$ is the normal smoothness operator. This operator * is linear and continuous from $H^{-\frac{1}{2}}\left(\Gamma_{C}\right)$ to $L^{2}\left(\Gamma_{C}\right)$. Usually, it is taken as a convolution, $\tau^{*}=\omega * \tau, \tau \in H^{-1 / 2}\left(\Gamma_{C}\right)$, where $\omega \in \mathcal{D}(-\delta, \delta), \int_{-\delta}^{\delta} \omega=1$, with $\delta \in \mathbb{R}, \delta>0$, but other choices can be made (see Badea and Krause 2012, for instance). We suppose that $u_{0} \in\left(H^{\frac{1}{2}}\left(\Gamma_{D}\right)\right)^{d}$, and define the sets of statically admissible fields:

$$
\begin{aligned}
H & =H_{\text {div }}(\Omega)=\left\{\tau=\left(\tau_{i j}\right)_{1 \leq i, j \leq d}: \tau_{i j}=\tau_{j i}, 1 \leq i, j \leq d, \tau \in\left(L^{2}(\Omega)\right)^{d \times d}, \operatorname{div} \tau \in\left(L^{2}(\Omega)\right)^{d}\right\} \\
H_{f, g} & =\left\{\sigma \in H ; \operatorname{div} \sigma+f=0 \text { in } \Omega, \sigma n=g \text { on } \Gamma_{F}\right\} \\
\Sigma_{\tau} & =\left\{\sigma \in H_{f, g} ; \sigma_{N} \leq 0,\left|\sigma_{T}\right| \leq-\mu \tau_{N} \text { on } \Gamma_{C}\right\}
\end{aligned}
$$

where $\tau \in\left(L^{2}\left(\Gamma_{C}\right)\right)^{d}$.

The case of the Tresca friction is a particular case of the last equation in (2.2), where the normal stress $\sigma_{N}^{*}$ is replaced by a given $\eta \in\left(L^{2}\left(\Gamma_{C}\right)\right)^{d}$. Note that the solution of the contact problem with Coulomb friction can be obtained by a fixed point procedure in which we solve a contact problem with Tresca friction at each iteration Licht et al. (1991). The stress field variational formulation of (P) with Tresca friction law is (see Telega 1991, for instance):

Problem $\left(\mathbf{P}_{\mathbf{s}}\right)$. Find the stress field $\sigma: \Omega \rightarrow H_{f, g}$ such that

$$
\left\{\begin{array}{l}
\sigma \in \Sigma_{\eta} \\
s(\sigma, \tau-\sigma) \geq l(\tau-\sigma) \quad \text { for any } \tau \in \Sigma_{\eta}
\end{array}\right.
$$


with $s(\sigma, \tau)=\int_{\Omega} S \sigma: \tau \mathrm{d} x$ and $l(\tau)=\int_{\Gamma_{D}} u_{0} \cdot \tau n \mathrm{~d} s$. From the properties of the operator $S$, we can conclude that the bilinear form $s$ is symmetric and there exist two constants $\alpha, \beta>0$ such that, for any $\sigma, \tau \in H_{f, g}$, we have

$$
\begin{gathered}
\alpha\|\tau-\sigma\|_{H_{\mathrm{div}}(\Omega)}^{2} \leq s(\tau-\sigma, \tau-\sigma) \quad \text { and } \\
s(\sigma, \tau) \leq \beta\|\sigma\|_{H_{\mathrm{div}}(\Omega)}\|\tau\|_{H_{\mathrm{div}}(\Omega)}
\end{gathered}
$$

where

$$
\|\tau\|_{H_{\text {div }}(\Omega)}^{2}=\int_{\Omega}(\tau: \tau+\operatorname{div} \tau \cdot \operatorname{div} \tau) .
$$

Also, there exists $M>0$ such that

$$
l(\tau) \leq M\|\tau\|_{H_{\operatorname{div}}(\Omega)}
$$

for any $\tau \in H$. In view of the above properties (2.5) and (2.6) of the bilinear form $s$ and of the functional $l$, we notice that problem (2.4) has a unique solution $\sigma \in \Sigma_{\eta}$ which also minimizes the functional $I(\tau)=\frac{1}{2} s(\tau, \tau)-l(\tau)$ over all $\tau \in \Sigma_{\eta}$. These properties of $s$ and $l$ are also needed in the convergence proof of the Schwarz method.

\section{Schwarz algorithm}

Let us consider a domain decomposition of $\Omega$,

$$
\Omega=\cup_{i=1}^{m} \Omega_{i}
$$

with an overlapping parameter $\delta$, and we assume that it has the property

$$
\operatorname{meas}\left(\partial\left(\Omega_{i} \cap\left(\cup_{j=i+1}^{m} \Omega_{j}\right)\right) \cap \Gamma_{D}\right)>0 \text { for any } i=1, \ldots, m-1
$$

This is an existence condition for the solutions of some auxiliary problems used in the proof of Lemma 3.1 to prove that Assumption 3.1 below is satisfied. We associate to this decomposition the spaces

$$
\begin{aligned}
H_{0,0}^{i} & =\left\{\sigma \in H_{\operatorname{div}}(\Omega): \operatorname{div} \sigma=0 \text { in } \Omega_{i}, \sigma=0 \text { on } \Omega \backslash \Omega_{i}\right. \\
\sigma_{\mid \Omega_{i}} n & \left.=0 \text { on }\left(\Omega \cap \partial \Omega_{i}\right) \cup\left(\Gamma_{F} \cap \partial \Omega_{i}\right)\right\}, \quad i=1, \ldots, m
\end{aligned}
$$

We point out that the elements of $H_{0,0}^{i}$ are elements of $H_{0,0}(\Omega)$ which vanish on $\Omega \backslash \Omega_{i}$. We introduce the Schwarz algorithm, written as subspace correction method, for the solution of problem (2.4) as

Algorithm 3.1 We start the algorithm with an arbitrary $\sigma^{0} \in \Sigma_{\eta}$. At iteration $n+1$, having $\sigma^{n} \in \Sigma_{\eta}, n \geq 0$, we compute sequentially for $i=1, \ldots, m$, the local corrections $\sigma_{i}^{n+1}$ as the solution of the variational inequalities

$$
\begin{aligned}
& \sigma_{i}^{n+1} \in H_{0,0}^{i}, \sigma^{n+\frac{i-1}{m}}+\sigma_{i}^{n+1} \in \Sigma_{\eta}: s\left(\sigma^{n+\frac{i-1}{m}}+\sigma_{i}^{n+1}, \sigma_{i}-\sigma_{i}^{n+1}\right) \geq l\left(\sigma_{i}-\sigma_{i}^{n+1}\right) \\
& \text { for any } \sigma_{i} \in H_{0,0}^{i}, \sigma^{n+\frac{i-1}{m}}+\sigma_{i} \in \Sigma_{\eta}
\end{aligned}
$$

and then we update $\sigma^{n+\frac{i}{m}}=\sigma^{n+\frac{i-1}{m}}+\sigma_{i}^{n+1}$. 
In this algorithm, we can take $l\left(\sigma_{i}-\sigma_{i}^{n+1}\right)=\int_{\Gamma_{D}} u_{0} \cdot\left(\sigma_{i}-\sigma_{i}^{n+1}\right) n d s$ in the place of $\int_{\Gamma_{D} \cap \partial \Omega_{i}} u_{0} \cdot\left(\sigma_{i}-\sigma_{i}^{n+1}\right) n \mathrm{~d} s$ because $\sigma_{i}-\sigma_{i}^{n+1}$ vanishes outside $\Omega_{i}$. The convergence of the above algorithm is obtained from a more general convergence result in Badea (2003) given for algorithms in a reflexive Banach space. In this general framework, the convergence is proved by making an assumption and we have to show that it holds true in order to prove the convergence of a certain particular algorithm. In the case of our algorithm, this assumption is written below and the main result of the paper is to show that it is satisfied for the spaces introduced above. We point out that this assumption has only a theoretical value, the fact that it is satisfied is a sufficient condition for the convergence of the algorithm, but it is not used in the practical application of the algorithm.

Assumption 3.1 There exists a constant $C_{0}$ such that for any $\sigma, \tau \in \Sigma_{\eta}$ and $\sigma_{i} \in H_{0,0}^{i}$, $\sigma+\sum_{j=1}^{i} \sigma_{j} \in \Sigma_{\eta}, i=1, \ldots, m$, there exist $\tau_{i} \in H_{0,0}^{i}, i=1, \ldots, m$, satisfying

$$
\begin{gathered}
\sigma+\sum_{j=1}^{i-1} \sigma_{j}+\tau_{i} \in \Sigma_{\eta} \quad \text { for } i=1, \ldots, m \\
\tau-\sigma=\sum_{i=1}^{m} \tau_{i} \\
\sum_{i=1}^{m}\left\|\tau_{i}\right\|_{H_{\mathrm{div}}(\Omega)}^{2} \leq C_{0}\left(\|\tau-\sigma\|_{H_{\mathrm{div}}(\Omega)}^{2}+\sum_{i=1}^{m}\left\|\sigma_{i}\right\|_{H_{\mathrm{div}}(\Omega)}^{2}\right) .
\end{gathered}
$$

We have the following result concerning the convergence of the algorithm.

Theorem 3.1 We suppose that Assumption 3.1 holds. Then, if $\sigma$ is the solution of problem (2.4) and $\sigma^{n}, n \geq 0$, are its approximations obtained from Algorithm 3.1, we have the following error estimation

$$
\left\|\sigma^{n}-\sigma\right\|_{H_{\mathrm{div}}(\Omega)}^{2} \leq C\left(\frac{\tilde{C}}{\tilde{C}+1}\right)^{n}
$$

where $C$ and $\tilde{C}$ are two positive constants, $C$ depends on $\sigma, \sigma^{0}$ and $\alpha$, and $\tilde{C}$ depends on the constants $\alpha, \beta$, the number $m$ of subdomains, and is an increasing function of the constant $C_{0}$ introduced in Assumption 3.1.

Proof Since the bilinear form $s$ and the linear functional $l$ satisfy (2.5) and (2.6), we can apply Theorem 2 in Badea (2003) to get the convergence of Algorithm 3.1 and the error estimation. Constant $\tilde{C}$ can be taken as

$$
\tilde{C}=\frac{2 \beta m}{\alpha(1-\kappa)}\left[1+2 C_{0}+\frac{2 \beta m}{\alpha \kappa} C_{0}^{2}\right],
$$

where $0<\kappa<1$ is chosen such that $\tilde{C}$ has a minimum value. Also, we can take

$$
C=\frac{2}{\alpha}\left[\frac{1}{2} s\left(\sigma^{0}, \sigma^{0}\right)+l\left(\sigma^{0}\right)-\frac{1}{2} s(\sigma, \sigma)-l(\sigma)\right] .
$$

where $\sigma^{0}$ is the starting approximation in algorithms and $\sigma$ is the solution of problem (2.4).

Constants $\alpha$ and $\beta$ come from problem (2.4) and are given in (2.5). Also, the number $m$ of the subspaces can be associated with the number of colors needed to mark the subdomains 
such that the subdomains with the same color do not intersect with each other. Since, in general, this number of colors depends on the dimension of the Euclidean space where the domain lies (see Toselli and Widlund 2005, for instance), we can conclude that the convergence rate of the algorithms essentially depends on the constant $C_{0}$. Moreover, $\tilde{C}$ and the convergence rate, too, are increasing functions of $C_{0}$. Indeed, by a simple calculus, we get from (3.13) that

$$
\tilde{C}=\frac{2 \beta m}{\alpha}\left(\sqrt{\frac{2 \beta m}{\alpha} C_{0}^{2}+2 C_{0}+1}+\sqrt{\frac{2 \beta m}{\alpha} C_{0}^{2}}\right)^{2}
$$

In the following, we prove that Assumption 3.1 holds and estimate this constant $C_{0}$.

To prove that this assumption holds, we associate to the decomposition (3.7) of $\Omega$, some functions $\theta_{i} \in H^{1}(\Omega), i=1, \ldots, m$, such that

$$
0 \leq \theta_{i} \leq 1 \text { on } \Omega, \theta_{i}=0 \text { on } \cup_{j=i+1}^{m} \Omega_{j} \backslash \Omega_{i} \text {, and } \theta_{i}=1 \text { on } \Omega_{i} \backslash \cup_{j=i+1}^{m} \Omega_{j} \text {. }
$$

Since the overlapping size of the domain decomposition is $\delta$, the above functions $\theta_{i}$ can be chosen to satisfy

$$
\left|\partial_{x_{k}} \theta_{i}\right| \leq C / \delta, \quad \text { a.e. in } \Omega, \quad \text { for any } k=1, \ldots, d,
$$

where $C$ is a constant independent of the domain decomposition.

Now, let us consider $\sigma \in \Sigma_{\eta}, \sigma_{i} \in H_{0,0}^{i}$ such that $\sigma+\sum_{j=1}^{i} \sigma_{j} \in \Sigma_{\eta}, i=1, \ldots, m$, and let $\tau$ be another element in $\Sigma_{\eta}$. Using the functions $\theta_{i}, i=1, \ldots, m$ defined in (3.15) and (3.16), we first define $\tau_{i}, i=1, \ldots, m$.

For $i=1$, we consider $u_{1} \in H^{1}\left(\Omega_{1} \cap\left(\cup_{j=2}^{m} \Omega_{j}\right)\right), u_{1}=0$ on $\partial\left(\Omega_{1} \cap\left(\cup_{j=2}^{m} \Omega_{j}\right)\right) \cap \Gamma_{D}$, the solution of the problem

$$
\begin{aligned}
& \int_{\Omega_{1} \cap\left(\cup_{j=2}^{m} \Omega_{j}\right)} A \varepsilon\left(u_{1}\right): \varepsilon\left(v_{1}\right)+\int_{\partial\left(\Omega_{1} \cap\left(\cup_{j=2}^{m} \Omega_{j}\right) \cap \cap \Gamma_{C}\right.}\left[\theta_{1}(\tau-\sigma)+\left(1-\theta_{1}\right) \sigma_{1}\right] n \cdot v_{1} \\
& \quad+\int_{\partial\left(\cup_{j=2}^{m} \Omega_{j}\right) \cap \Omega_{1}}(\tau-\sigma) n \cdot v_{1}=0
\end{aligned}
$$

for any $v_{1} \in H^{1}\left(\Omega_{1} \cap\left(\cup_{j=2}^{m} \Omega_{j}\right)\right), v_{1}=0$ on $\partial\left(\Omega_{1} \cap\left(\cup_{j=2}^{m} \Omega_{j}\right)\right) \cap \Gamma_{D}$, and define

$$
\tau_{1}= \begin{cases}\tau-\sigma & \text { on } \Omega_{1} \backslash\left(\cup_{j=2}^{m} \Omega_{j}\right) \\ A \varepsilon\left(u_{1}\right) & \text { on } \Omega_{1} \cap\left(\cup_{j=2}^{m} \Omega_{j}\right) \\ 0 & \text { on }\left(\cup_{j=2}^{m} \Omega_{j}\right) \backslash \Omega_{1}\end{cases}
$$

For $i=2$, we make the same construction as above by taking $\tau-\sigma-\tau_{1}$ in the place of $\tau-\sigma, \Omega_{2}$ in the place of $\Omega_{1}$ and $\cup_{j=2}^{m} \Omega_{j}$ in the place of $\Omega=\cup_{j=1}^{m} \Omega_{j}$. In this way, we look for the solution $u_{2} \in H^{1}\left(\Omega_{2} \cap\left(\cup_{j=3}^{m} \Omega_{j}\right)\right), u_{2}=0$ on $\partial\left(\Omega_{2} \cap\left(\cup_{j=3}^{m} \Omega_{j}\right)\right) \cap \Gamma_{D}$, the solution of the problem 


$$
\begin{aligned}
& \int_{\Omega_{2} \cap\left(\cup_{j=3}^{m} \Omega_{j}\right)} A \varepsilon\left(u_{2}\right): \varepsilon\left(v_{2}\right)+\int_{\partial\left(\Omega_{2} \cap\left(\cup_{j=3}^{m} \Omega_{j}\right)\right) \cap \Gamma_{C}}\left[\theta_{2}\left(\tau-\sigma-\tau_{1}\right)+\left(1-\theta_{2}\right) \sigma_{1}\right] n \cdot v_{2} \\
& \quad+\int_{\partial\left(\cup_{j=3}^{m} \Omega_{j}\right) \cap \Omega_{2}}\left(\tau-\sigma-\tau_{1}\right) n \cdot v_{2}=0
\end{aligned}
$$

for any $v_{2} \in H^{1}\left(\Omega_{2} \cap\left(\cup_{j=3}^{m} \Omega_{j}\right)\right), v_{2}=0$ on $\partial\left(\Omega_{2} \cap\left(\cup_{j=3}^{m} \Omega_{j}\right)\right) \cap \Gamma_{D}$, and define

$$
\tau_{2}= \begin{cases}0 & \text { on } \Omega_{1} \backslash\left(\cup_{j=2}^{m} \Omega_{j}\right) \\ \tau-\sigma-\tau_{1} & \text { on } \Omega_{2} \backslash\left(\cup_{j=3}^{m} \Omega_{j}\right) \\ A \varepsilon\left(u_{2}\right) & \text { on } \Omega_{2} \cap\left(\cup_{j=3}^{m} \Omega_{j}\right) \\ 0 & \text { on }\left(\cup_{j=3}^{m} \Omega_{j}\right) \backslash \Omega_{2}\end{cases}
$$

For a certain $3 \leq i \leq m-1$, we consider $\tau-\sigma-\tau_{1}-\cdots-\tau_{i-1}$, the domains $\Omega_{i}$ and $\cup_{j=i+1}^{m} \Omega_{j}$ and $u_{i} \in H^{1}\left(\Omega_{i} \cap\left(\cup_{j=i+1}^{m} \Omega_{j}\right)\right), u_{i}=0$ on $\partial\left(\Omega_{i} \cap\left(\cup_{j=i+1}^{m} \Omega_{j}\right)\right) \cap \Gamma_{D}$, the solution of the problem

$$
\begin{gathered}
\int_{\Omega_{i} \cap\left(\cup_{j=i+1}^{m} \Omega_{j}\right)} A \varepsilon\left(u_{i}\right): \varepsilon\left(v_{i}\right)+\int_{\partial\left(\Omega_{i} \cap\left(\cup_{j=i+1}^{m} \Omega_{j}\right)\right) \cap \Gamma_{C}}\left[\theta_{i}\left(\tau-\sigma-\tau_{1}-\cdots-\tau_{i-1}\right)\right. \\
\left.+\left(1-\theta_{i}\right) \sigma_{i}\right] n \cdot v_{i}+\int_{\partial\left(\cup_{j=i+1}^{m} \Omega_{j}\right) \cap \Omega_{i}}\left(\tau-\sigma-\tau_{1}-\cdots-\tau_{i-1}\right) n \cdot v_{i}=0
\end{gathered}
$$

for any $v_{i} \in H^{1}\left(\Omega_{i} \cap\left(\cup_{j=i+1}^{m} \Omega_{j}\right)\right), v_{i}=0$ on $\partial\left(\Omega_{i} \cap\left(\cup_{j=i+1}^{m} \Omega_{j}\right)\right) \cap \Gamma_{D}$, and define

$$
\tau_{i}= \begin{cases}0 & \text { on }\left(\cup_{j=1}^{i-1} \Omega_{j}\right) \backslash\left(\cup_{j=i}^{m} \Omega_{j}\right) \\ \tau-\sigma-\tau_{1}-\cdots-\tau_{i-1} & \text { on } \Omega_{i} \backslash\left(\cup_{j=i+1}^{m} \Omega_{j}\right) \\ A \varepsilon\left(u_{i}\right) & \text { on } \Omega_{i} \cap\left(\cup_{j=i+1}^{m} \Omega_{j}\right) \\ 0 & \text { on }\left(\cup_{j=i+1}^{m} \Omega_{j}\right) \backslash \Omega_{i}\end{cases}
$$

Finally, we define

$$
\tau_{m}=\tau-\sigma-\sum_{j=1}^{m-1} \tau_{j}
$$

The construction of these $\tau_{i}, i=1, \ldots, m$, concerns only the verification of the assumptions, but not the application of the algorithm. We have the following result concerning the above defined $\tau_{1}, \ldots, \tau_{m}$.

Lemma 3.1 Let $\tau_{1}, \ldots, \tau_{m}$ be defined in (3.17)-(3.23). Then,

$$
\begin{gathered}
\tau_{i} \in H_{0,0}^{i} \quad \text { for } i=1, \ldots, m \\
\tau-\sigma-\sum_{j=1}^{i} \tau_{j}=0 \text { on } \Omega \backslash\left(\cup_{j=i+1}^{m} \Omega_{j}\right) \text { for } i=1, \ldots, m-1 \\
\sigma+\tau_{1} \in \Sigma_{\eta} \text { and } \sigma+\sum_{j=1}^{i-1} \sigma_{j}+\tau_{i} \in \Sigma_{\eta} \quad \text { for } i=2, \ldots, m \\
\tau-\sum_{j=1}^{i} \tau_{j}+\sum_{j=1}^{i} \sigma_{j} \in \Sigma_{\eta} \quad \text { for } i=1, \ldots, m-1
\end{gathered}
$$


Proof We notice that, in view of the condition (3.8) on the domain decomposition, Eqs. (3.17), (3.19) and (3.21) have unique solutions. The proof of lemma is made by induction.

(a) For $i=1$, from (3.17), we get $\operatorname{div} A \varepsilon\left(u_{1}\right)=0$ on $\Omega_{1} \cap\left(\cup_{j=2}^{m} \Omega_{j}\right)$ and $A \varepsilon\left(u_{1}\right) n=$ $(\tau-\sigma) n$ on $\partial\left(\cup_{j=2}^{m} \Omega_{j}\right) \cap \Omega_{1}$. Since $\operatorname{div}(\tau-\sigma)=0$ on $\Omega_{1} \backslash\left(\cup_{j=2}^{m} \Omega_{j}\right)$, we get from (3.18) that

$$
\tau_{1 \mid \Omega_{1}} \in H_{\text {div }}\left(\Omega_{1}\right) \text { and } \operatorname{div}\left(\tau_{1 \mid \Omega_{1}}\right)=0 \text { on } \Omega_{1}
$$

Since $(\tau-\sigma) n=0$ on $\Gamma_{F}$ and, in view of (3.17), we have $A \varepsilon\left(u_{1}\right) n=0$ on $\partial\left(\Omega_{1} \cap\right.$ $\left.\left(\cup_{j=2}^{m} \Omega_{j}\right)\right) \cap \Gamma_{F}$, we get

$$
\tau_{1 \mid \Omega_{1}} n=0 \text { on } \partial \Omega_{1} \cap \Gamma_{F}
$$

Also, we get from (3.17) that $A \varepsilon\left(u_{1}\right) n=0$ on $\partial \Omega_{1} \cap\left(\cup_{j=2}^{m} \Omega_{j}\right)$, or

$$
\tau_{1 \mid \Omega_{1}} n=0 \text { on } \partial \Omega_{1} \cap \Omega
$$

and therefore, the extension of $\tau_{1 \mid \Omega_{1}}$ with zero in $\Omega \backslash \Omega_{1}$ is an element of $H_{0,0}(\Omega)$. Consequently, (3.24) holds for $\tau_{1}$ defined in (3.18).

From the definition of $\tau_{1}$ we get that $\tau-\sigma-\tau_{1}=0$ on $\Omega_{1} \backslash\left(\cup_{j=2}^{m} \Omega_{j}\right)$, and consequently, (3.25) holds for $i=1$.

Since $\tau_{1} \in H_{0,0}^{1}$ and $\tau, \sigma \in H_{f, g}$, we get

$$
\sigma+\tau_{1} \in H_{f, g}
$$

On $\partial\left(\Omega_{1} \cap\left(\cup_{j=2}^{m} \Omega_{j}\right)\right) \cap \Gamma_{C}$, using again (3.17), we have

$$
\left(\sigma+\tau_{1}\right) n=\left(\sigma+A \varepsilon\left(u_{1}\right)\right) n=\left[\theta_{1} \tau+\left(1-\theta_{1}\right)\left(\sigma+\sigma_{1}\right)\right] n
$$

Since $\tau, \sigma, \sigma+\sigma_{1} \in \Sigma_{\eta}$, in view of the above equation, we have

$$
\left(\sigma+\tau_{1}\right)_{N} \leq 0 \text { and }\left|\left(\sigma+\tau_{1}\right)_{T}\right| \leq-\mu \eta_{N} \text { on } \Gamma_{C}
$$

In this way, we get that (3.26) holds for $i=1$.

In a similar way, we prove that (3.27) holds for $i=1$. First, we see that

$$
\tau-\tau_{1}+\sigma_{1} \in H_{f, g}
$$

Also, on $\partial\left(\Omega_{1} \cap\left(\cup_{j=2}^{m} \Omega_{j}\right)\right) \cap \Gamma_{C}$, we have

$$
\left(\tau-\tau_{1}+\sigma_{1}\right) n=\left(\tau-A \varepsilon\left(u_{1}\right)+\sigma_{1}\right) n=\left[\theta_{1}\left(\sigma+\sigma_{1}\right)+\left(1-\theta_{1}\right) \tau\right] n
$$

and, from here and the fact that $\tau, \sigma, \sigma+\sigma_{1} \in \Sigma_{\eta}$, we get

$$
\left(\tau-\tau_{1}+\sigma_{1}\right)_{N} \leq 0 \text { and }\left|\left(\tau-\tau_{1}+\sigma_{1}\right)_{T}\right| \leq-\mu \eta_{N} \text { on } \Gamma_{C}
$$

Consequently, we get that (3.27) holds for $i=1$.

(b) For $i=2$, let us write

$$
\tilde{\sigma}=\sigma+\sigma_{1} \text { and } \tilde{\tau}=\tau-\tau_{1}+\sigma_{1}
$$

First, from (3.25) for $i=1$, we get that

$$
\tilde{\tau}-\tilde{\sigma}=\tau-\sigma-\tau_{1}=0 \text { on } \Omega \backslash\left(\cup_{i=2}^{m} \Omega_{i}\right)
$$

Then, we reduce the case of $i=2$ to that of $i=1$ by considering the decomposition $\Omega_{2}, \ldots, \Omega_{m}$ of $\cup_{j=2}^{m} \Omega_{j}$ instead of the decomposition $\Omega_{1}, \ldots, \Omega_{m}$ of $\cup_{j=1}^{m} \Omega_{j}$, and $\tilde{\sigma}$ and $\tilde{\tau}$ in the place of $\sigma$ and $\tau$, respectively. 
From (3.29) and the definition of $\tau_{2}$ in (3.20), we get that if we prove that $\tau_{2 \mid \cup j=2}^{m} \Omega_{j}$ belongs to $H_{0,0}^{2}$ corresponding to $\Omega_{2}$ in the decomposition $\Omega_{2}, \ldots, \Omega_{m}$ of $\cup_{j=2}^{m} \Omega_{j}$, then $\tau_{2}$ belongs to $H_{0,0}^{2}$ corresponding to $\Omega_{2}$ in the decomposition $\Omega_{1}, \ldots, \Omega_{m}$ of $\cup_{j=1}^{m} \Omega_{j}$, i.e. (3.24) is satisfied for $i=2$.

We see that by replacing $\tilde{\sigma}$ and $\tilde{\tau}$ in the definition of $\tau_{2}$ given in (3.19) and (3.20), we get that $\tau_{2 \mid \cup \cup_{j=2}^{m} \Omega_{j}}$ is identically defined as $\tau_{1}$ in (3.17) and (3.18) where $\sigma$ and $\tau$ are replaced by $\tilde{\sigma}$ and $\tilde{\tau}$, respectively. Besides this, in view of the conditions in Assumption 3.1, we have $\tilde{\sigma}, \tilde{\sigma}+\sigma_{2} \in \Sigma_{\eta}$. Also, from (3.27) with $i=1$, we have $\tilde{\tau} \in \Sigma_{\eta}$. Consequently, repeating the reasoning from $i=1$ in the new context, we get that, for the decomposition $\Omega_{2}, \ldots, \Omega_{m}$ of $\cup_{j=2}^{m} \Omega_{j}, \tau_{2 \mid \cup_{j=2}^{m} \Omega_{j}}$ satisfies (3.24)-(3.27) from $i=1$ with $\sigma$ and $\tau$ replaced by $\tilde{\sigma}$ and $\tilde{\tau}$, respectively. Now, replacing $\tilde{\sigma}$ and $\tilde{\tau}$ in these equations with their values given in (3.28) and taking into account (3.29), we get that (3.24)-(3.27) hold for $i=2$.

(c) For a given $3 \leq i \leq m-1$ we define

$$
\tilde{\sigma}=\sigma+\sum_{j=1}^{i-1} \sigma_{j} \text { and } \tilde{\tau}=\tau-\sum_{j=1}^{i-1} \tau_{j}+\sum_{j=1}^{i-1} \sigma_{j}
$$

and, with the above reasoning, we get that (3.24)-(3.27) for that $i$.

(d) For $i=m$, we consider $\tau_{m}$ defined in (3.23). Since $\tau, \sigma, \sigma_{1}, \ldots, \sigma_{m-1} \in H_{\text {div }}(\Omega)$, it follows that $\tau_{m} \in H_{\mathrm{div}}(\Omega)$. Moreover, from (3.25) for $i=m-1$, we get that $\tau_{m}=0$ on $\Omega \backslash \Omega_{m}$. Besides that, since $\tau-\sigma \in H_{0,0}$ and, for $i=1, \ldots, m-1, \tau_{i} \in H_{0,0}^{i}$, we get

$$
\tau_{m} \in H_{0,0}^{m},
$$

i.e. (3.24) holds for $i=m$.

Finally, using (3.27) for $i=m-1$, we get

$$
\sigma+\sum_{j=1}^{m-1} \sigma_{j}+\tau_{m}=\tau+\sum_{j=1}^{m-1} \sigma_{j}-\sum_{j=1}^{m-1} \tau_{j} \in \Sigma_{\eta}
$$

i.e. (3.26) holds for $i=m$.

Now, we can prove

Proposition 3.1 Assumption 3.1 holds true with

$$
C_{0}=m \sum_{j=0}^{m-1} C_{m \delta}^{j}
$$

where

$$
C_{m \delta}=C m\left(1+\frac{d}{\delta^{2}}\right)
$$

constant $C$ being independent of the domain decomposition.

Proof Let us consider $\tau, \sigma \in \Sigma_{\eta}, \sigma_{i} \in H_{0,0}^{i}$ such that $\sigma+\sum_{j=1}^{i} \sigma_{j} \in \Sigma_{\eta}$, and let $\tau_{1}, \ldots, \tau_{m}$ be defined in (3.17)-(3.23). We shall use Lemma 3.1 to prove that (3.10) and (3.11) hold. First, (3.24) shows that $\tau_{i} \in H_{0,0}^{i}$ for $i=1, \ldots, m$. Then, (3.26) proves (3.10), and finally, (3.23) is (3.11).

We now prove that (3.12) holds with $C_{0}$ given in (3.30), and we first evaluate the norms of $\tau_{i}, i=1, \ldots, m$. 
(a) For $i=1$, by replacing $v_{1}$ with $u_{1}$ in (3.17), and using the trace theorems in $H^{1}$ and $H_{\text {div }}$ (see Theorem 2.5, page 27, in Girault and Raviart 1986 for the vectorial case, for instance), we get

$$
\begin{aligned}
\int_{\Omega_{1} \cap\left(\cup_{j=2}^{m} \Omega_{j}\right)} A \varepsilon\left(u_{1}\right): \varepsilon\left(u_{1}\right)= & -\int_{\partial\left(\Omega_{1} \cap\left(\cup_{j=2}^{m} \Omega_{j}\right)\right) \cap \Gamma_{C}}\left[\theta_{1}(\tau-\sigma)+\left(1-\theta_{1}\right) \sigma_{1}\right] n \cdot u_{1} \\
& -\int_{\partial\left(\cup_{j=2}^{m} \Omega_{j}\right) \cap \Omega_{1}}(\tau-\sigma) n \cdot u_{1} \\
= & -\int_{\partial\left(\Omega_{1} \cap\left(\cup_{j=2}^{m} \Omega_{j}\right)\right) \cap\left(\Gamma_{C} \cup \Omega_{1}\right)}\left[\theta_{1}(\tau-\sigma)+\left(1-\theta_{1}\right) \sigma_{1}\right] n \cdot u_{1} \\
\leq & \left\|\theta_{1}(\tau-\sigma) n+\left(1-\theta_{1}\right) \sigma_{1} n\right\|_{H^{-1 / 2}\left(\partial\left(\Omega_{1} \cap\left(\cup_{j=2}^{m} \Omega_{j}\right)\right)\right)} \\
& \cdot\left\|u_{1}\right\|_{H^{1 / 2}\left(\partial\left(\Omega_{1} \cap\left(\cup_{j=2}^{m} \Omega_{j}\right)\right)\right)} \leq C \| \theta_{1}(\tau-\sigma) \\
& +\left(1-\theta_{1}\right) \sigma_{1}\left\|_{H_{\mathrm{div}}\left(\Omega_{1} \cap\left(\cup_{j=2}^{m} \Omega_{j}\right)\right)}\right\| u_{1} \|_{H^{1}\left(\Omega_{1} \cap\left(\cup_{j=2}^{m} \Omega_{j}\right)\right)}
\end{aligned}
$$

Above, we have used the fact that $\theta_{1}=1$ on $\partial\left(\cup_{j=2}^{m} \Omega_{j}\right) \cap \Omega_{1}=\partial\left(\Omega_{1} \cap\left(\cup_{j=2}^{m} \Omega_{j}\right)\right) \cap \Omega_{1}$. Constant $C$ can be taken depending only of $\Omega$, independently of the domain decomposition. From now on, we denote by $C$ a generic constant with this property. Finally, we have written, for instance, $\|\cdot\|_{H^{1}\left(\Omega_{1} \cap\left(\cup_{j=2}^{m} \Omega_{j}\right)\right)}$ in the place of $\|\cdot\|_{H^{1}\left(\Omega_{1} \cap\left(\cup_{j=2}^{m} \Omega_{j}\right)\right)^{d}}$. The superscripts $d$ or $d \times d$ in the notation of the norms will also be omitted in the following. In view of (3.8), we have (see Théorème 3.3, p. 115, in Duvaut and Lions 1972, for instance)

$$
\left\|u_{1}\right\|_{H^{1}\left(\Omega_{1} \cap\left(\cup_{j=2}^{m} \Omega_{j}\right)\right)}^{2} \leq C \int_{\Omega_{1} \cap\left(\cup_{j=2}^{m} \Omega_{j}\right)} A \varepsilon\left(u_{1}\right): \varepsilon\left(u_{1}\right)
$$

Also, using the properties of $S$, we have

$$
\begin{aligned}
\gamma\left\|A \varepsilon\left(u_{1}\right)\right\|_{L^{2}\left(\Omega_{1} \cap\left(\cup_{j=2}^{m} \Omega_{j}\right)\right)}^{2} \leq & \left(S\left(A \varepsilon\left(u_{1}\right)\right), A \varepsilon\left(u_{1}\right)\right)_{L^{2}\left(\Omega_{1} \cap\left(\cup_{j=2}^{m} \Omega_{j}\right)\right)} \\
& \times \int_{\Omega_{1} \cap\left(\cup_{j=2}^{m} \Omega_{j}\right)} A \varepsilon\left(u_{1}\right): \varepsilon\left(u_{1}\right)
\end{aligned}
$$

From the above three equations, we get

$$
\left\|A \varepsilon\left(u_{1}\right)\right\|_{L^{2}\left(\Omega_{1} \cap\left(\cup_{j=2}^{m} \Omega_{j}\right)\right)} \leq C\left\|\theta_{1}(\tau-\sigma)+\left(1-\theta_{1}\right) \sigma_{1}\right\|_{H_{\mathrm{div}}\left(\Omega_{1} \cap\left(\cup_{j=2}^{m} \Omega_{j}\right)\right)}
$$

and since $\operatorname{div}\left(A \varepsilon\left(u_{1}\right)\right)=0$, we have

$$
\left\|A \varepsilon\left(u_{1}\right)\right\|_{H_{\mathrm{div}}\left(\Omega_{1} \cap\left(\cup_{j=2}^{m} \Omega_{j}\right)\right)} \leq C\left\|\theta_{1}(\tau-\sigma)+\left(1-\theta_{1}\right) \sigma_{1}\right\|_{H_{\mathrm{div}}\left(\Omega_{1} \cap\left(\cup_{j=2}^{m} \Omega_{j}\right)\right)}
$$

Moreover, in view of (3.18) and since $\theta_{1}=1$ on $\left.\Omega_{1} \backslash\left(\cup_{j=2}^{m} \Omega_{j}\right)\right)$, we get

$$
\left\|\tau_{1}\right\|_{H_{\mathrm{div}}(\Omega)} \leq C\left\|\theta_{1}(\tau-\sigma)+\left(1-\theta_{1}\right) \sigma_{1}\right\|_{H_{\mathrm{div}}\left(\Omega_{1}\right)}
$$

or, in view of (3.16) and taking into account that $\operatorname{div}(\tau-\sigma)=\operatorname{div} \sigma_{1}=0$ on $\Omega$, we get 


$$
\begin{aligned}
\left\|\tau_{1}\right\|_{H_{\text {div }}(\Omega)} & \leq C\left(1+\frac{d}{\delta^{2}}\right)^{\frac{1}{2}}\left[\|\tau-\sigma\|_{L^{2}\left(\Omega_{1}\right)}+\left\|\sigma_{1}\right\|_{L^{2}\left(\Omega_{1}\right)}\right] \\
& =C\left(1+\frac{d}{\delta^{2}}\right)^{\frac{1}{2}}\left[\|\tau-\sigma\|_{H_{\text {div }}\left(\Omega_{1}\right)}+\left\|\sigma_{1}\right\|_{H_{\text {div }}\left(\Omega_{1}\right)}\right] \\
& \leq C\left(1+\frac{d}{\delta^{2}}\right)^{\frac{1}{2}}\left[\|\tau-\sigma\|_{H_{\text {div }}(\Omega)}+\left\|\sigma_{1}\right\|_{H_{\text {div }}(\Omega)}\right]
\end{aligned}
$$

Consequently, we can write

$$
\left\|\tau_{1}\right\|_{H_{\mathrm{div}}(\Omega)}^{2} \leq C_{m \delta}\left[\|\tau-\sigma\|_{H_{\mathrm{div}}(\Omega)}^{2}+\left\|\sigma_{1}\right\|_{H_{\mathrm{div}}(\Omega)}^{2}\right]
$$

where $C_{m \delta}$ is given in (3.31).

(b) For $2 \leq i \leq m-1$, using the same arguments as above, we get

$$
\left\|\tau_{i}\right\|_{H_{\mathrm{div}}(\Omega)} \leq C\left\|\theta_{i}\left(\tau-\sigma-\sum_{j=1}^{i-1} \tau_{j}\right)+\left(1-\theta_{i}\right) \sigma_{i}\right\|_{H_{\mathrm{div}}\left(\Omega_{i}\right)}
$$

and

$$
\begin{aligned}
& \left\|\tau_{i}\right\|_{H_{\mathrm{div}}(\Omega)} \leq C\left(1+\frac{d}{\delta^{2}}\right)^{\frac{1}{2}}\left[\|\tau-\sigma\|_{H_{\mathrm{div}}\left(\Omega_{i}\right)}+\sum_{j=1}^{i-1}\left\|\tau_{j}\right\|_{H_{\mathrm{div}}\left(\Omega_{i}\right)}+\left\|\sigma_{i}\right\|_{H_{\mathrm{div}}\left(\Omega_{i}\right)}\right] \\
& \leq C\left(1+\frac{d}{\delta^{2}}\right)^{\frac{1}{2}}\left[\|\tau-\sigma\|_{H_{\mathrm{div}}(\Omega)}+\sum_{j=1}^{i-1}\left\|\tau_{j}\right\|_{H_{\mathrm{div}}(\Omega)}+\left\|\sigma_{i}\right\|_{H_{\mathrm{div}}(\Omega)}\right]
\end{aligned}
$$

Also, we can write

$$
\left\|\tau_{i}\right\|_{H_{\text {div }}(\Omega)}^{2} \leq C_{m \delta}\left[\|\tau-\sigma\|_{H_{\text {div }}(\Omega)}^{2}+\sum_{j=1}^{i-1}\left\|\tau_{j}\right\|_{H_{\text {div }}(\Omega)}^{2}+\left\|\sigma_{i}\right\|_{H_{\text {div }}(\Omega)}^{2}\right]
$$

(c) In view of (3.32) and (3.33), we can prove by induction that

$$
\sum_{j=1}^{i}\left\|\tau_{j}\right\|_{H_{\mathrm{div}}(\Omega)}^{2} \leq \sum_{j=1}^{i} C_{m \delta}^{j}\left[\|\tau-\sigma\|_{H_{\mathrm{div}}(\Omega)}^{2}+\left\|\sigma_{i+1-j}\right\|_{H_{\mathrm{div}}(\Omega)}^{2}\right]
$$

for $i=1, \ldots, m-1$. Now, from (3.23), we have

$$
\left\|\tau_{m}\right\|_{H_{\mathrm{div}}(\Omega)}^{2} \leq m\left[\|\tau-\sigma\|_{H_{\mathrm{div}}(\Omega)}^{2}+\sum_{j=1}^{m-1}\left\|\tau_{j}\right\|_{H_{\mathrm{div}}(\Omega)}^{2}\right]
$$

From these two last equations we get

$$
\sum_{i=1}^{m}\left\|\tau_{i}\right\|_{H_{\mathrm{div}}(\Omega)}^{2} \leq m \sum_{j=0}^{m-1} C_{m \delta}^{j}\|\tau-\sigma\|_{H_{\mathrm{div}}(\Omega)}^{2}+m \sum_{j=1}^{m-1} C_{m \delta}^{j}\left\|\sigma_{m-j}\right\|_{H_{\mathrm{div}}(\Omega)}^{2}
$$

and therefore, we can take constant $C_{0}$ as in (3.30). 
Summing up the results in Theorem 3.1 and Proposition 3.1, we get

Corollary 3.1 We assume that the domain decomposition (3.7) satisfies (3.8). Then, if $\sigma$ is the solution of problem (2.4) and $\sigma^{n}, n \geq 0$, are its approximations obtained from Algorithm 3.1, we have the following error estimation

$$
\left\|\sigma^{n}-\sigma\right\|_{H_{\mathrm{div}}(\Omega)}^{2} \leq C\left(\frac{\tilde{C}}{\tilde{C}+1}\right)^{n}
$$

where $\tilde{C}$ and $C$ are given in (3.13) and (3.14), respectively, and constant $C_{0}$ in $\tilde{C}$ is given in (3.30).

Remark 3.1 For problems in primal variables (see Badea 2006, for instance), the construction of the functions similar to $\tau_{i}$ in (3.17)-(3.23) is directly done by writing $\tau_{i}=\theta_{i}(\tau-\sigma-$ $\left.\sum_{j=1}^{i-1} \tau_{j}\right)+\left(1-\theta_{i}\right) \sigma$. In this case, using property (3.16), we obtain, without using the recursivity in the proof of Proposition 3.1, that

$$
\left\|\tau_{i}\right\|_{H_{\mathrm{div}}(\Omega)}^{2} \leq C_{m \delta}\left[\|\tau-\sigma\|_{H_{\mathrm{div}}(\Omega)}^{2}+\sum_{j=1}^{i}\left\|\sigma_{j}\right\|_{H_{\mathrm{div}}(\Omega)}^{2}\right]
$$

in the place of (3.33). The introduction of $A \varepsilon\left(u_{i}\right)$ in the definition of $\tau_{i}$ imposes the use of the recursivity, and consequently, $C_{0}$ in (3.30) depends on the powers of $C_{m \delta}$. However, as we already said, the number $m$ of the subdomains can be considered as being the minimal number of colors needed to mark the subdomains, and therefore, $C_{0}$ does not depend on the actual number of subdomains in the domain decomposition.

\section{Conclusions}

We have studied the convergence of the Schwarz method for contact problems with Tresca friction formulated in stress variables. In this dual variable, the problem is written as a variational inequality in the space $H_{\mathrm{div}}(\Omega), \Omega$ being the domain of the problem. The method is introduced as a subspace correction algorithm and we have used a general result in Badea (2003) which proves the convergence provided that an assumption is satisfied. The main result of this paper was to prove that the assumption made in the general convergence theorem is verified for our particular variational inequality. As in the case of the classical problems formulated in primal variables, we show that the method is geometrically convergent with a convergence rate which depends on the overlapping parameter of the domain decomposition.

Acknowledgments The authors acknowledge the support of the Laboratoire Européen Associé CNRS Franco-Roumain de Mathématiques et Modélisation, LEA Math-Mode, for this paper.

\section{References}

Alart P, Curnier A (1991) A mixed formulation for frictional contact problems prone to Newton like solution methods. Comput Methods Appl Mech Eng 92:353-375

Alart P, Lebon F (1995) Solution of frictional contact problems using ILU and coarse/fine preconditioners. Comput Mech 16:98-105

Badea L (2003) Convergence rate of a multiplicative Schwarz method for strongly nonlinear inequalities. In: Barbu V et al (eds) Analysis and optimization of differential systems. Kluwer Academic Publishers, Boston, pp 31-42 (also available from http://www.imar.ro/lbadea) 
Badea L (2006) Convergence rate of a Schwarz multilevel method for the constrained minimization of nonquadratic functionals. SIAM J Numer Anal 44(2):449-477

Badea L, Krause R (2012) One- and two-level Schwarz methods for inequalities of the second kind and their application to frictional contact. Numer Math 120(4):573-599

Bisegna P, Lebon F, Maceri F (2001) D-PANA : a convergent block-relaxation solution method for the discretized dual formulation of the Signorini-Coulomb contact problem. C R Math Acad Sci Paris 333:1053-1058

Bisegna P, Lebon F, Maceri F (2004) Relaxation procedures for solving Signorini-Coulomb contact problems. Adv Eng Softw 35:595-600

Cocou M, Pratt E, Raous M (1996) Formulation and approximation of quasistatic frictional contact. Int J Eng Sci 34:783-798

Cocu M (1984) Existence of solutions of Signorini problems with friction. Int J Eng Sci 22:567-575

Duvaut G, Lions JL (1972) Les inéquations en mécanique et en physique. Dunod, Paris

Girault V, Raviart P-A (1986) Finite element methods for Navier-Stokes equations. In: Springer series in computational mechanics, vol 5. Springer, New York

Kuss F, Lebon F (2009) Stress based finite element methods for solving contact problems: comparisons between various solution methods. Adv Eng Softw 40:697-706

Kuss F, Lebon F (2011) Error estimation and mesh adaptation for SignoriniCoulomb problems using E-FEM. Comput Struct 89:1148-1154

Lebon F (2003) Contact problems with friction: models and simulations. Simul Model Pract Theory 11:449463

Licht C, Pratt E, Raous M (1991) International series of numerical mathematics, vol 101. Remarks on a numerical method for unilateral contact including friction. Birkhauser, Basel, pp 129-143

Raous M, Chabrand P, Lebon F (1988) Numerical methods for frictional contact problems and applications. J Theor Appl Mech 7:111-128

Telega JJ (1991) Quasi-static Signorini's contact problem with friction and duality. Int Ser Numer Math 101:199-214

Toselli A, Widlund O (2005) Domain decomposition methods_-algorithms and theory. Springer, New York

Wriggers P (2002) Computational contact mechanics. Wiley, New York 